\title{
Random laser tailored by directional stimulated emission
}

\author{
M. Leonetti, ${ }^{1}$ C. Conti, ${ }^{2}$ and C. López ${ }^{1, *}$ \\ ${ }^{1}$ Instituto de Ciencia de Materiales de Madrid (CSIC), Cantoblanco, E-28049 Madrid, Spain \\ ${ }^{2}$ Research Center INFM-CNR, clo Universitá di Roma Sapienza, I-00185 Rome, Italy
}

(Received 13 October 2011; published 25 April 2012)

\begin{abstract}
The spectrum of a random laser (RL) may appear either as a set of sharp resonances or as a smooth line superimposed on the fluorescence. Recently, Leonetti, Conti, and Lopez [Nat. Photonics 5, 615 (2011)] accounted for this duality with the onset of a mode-locked regime that is triggered by the increase of both the number of activated modes and of the intermode interaction and is accompanied by a pulse shortening. Here we report an extensive review of the experimental approach used by Leonetti et al., including the sample preparation and the particulars of the setup. Here we describe also the way in which our approach allows us either to set the degree of interaction between modes or to have a certain degree of control over the effective set of resonances brought to lasing. Moreover we report an investigation on the spatial properties of the RL which brings further confirmation of the synchronization picture, whose physical origin is deepened.
\end{abstract}

DOI: 10.1103/PhysRevA.85.043841

PACS number(s): 42.55.Zz, 42.60.Fc

\section{INTRODUCTION}

The possibility lasing a scattering medium was predicted in the 1960s by Letokhov [1] and experimentally realized for bulk lasing material and scattering particles dispersed in liquid dye in the recent decades [2-4]. These pioneering experiments on random lasers (RL) have been characterized by a line-narrowed frequency spectrum (of the order of ten nanometers) on the top of the active medium fluorescence spectrum. This phenomenon, denoted as intensity feedback random lasers (IFRL), may be explained in the framework of diffusion approximation [5] in which all the properties of photon propagation are defined by the transport mean free path $\ell$. This approach, which neglects the wavelike nature of light, is particulary useful in different scattering regimes, where it may be used to predict particular properties of RL, such as fluctuations [6], or the appearance of spikes in the spectrum at random positions [7]. On the other hand, the diffusion approximation does not allow us to predict the existence of RL peaks that have been more recently measured in numerous experiments [8]. These peaks, which appear at a fixed wavelength and whose light is emitted from localized portions of the disordered structure, may be associated with the presence of Anderson localized states [9] or with highly scattering resonances dwelling in the disordered structures [10], which are commonly referred to as resonant feedback random lasers (RFRL). This kind of lasing emission has been observed in very strong scattering systems (zinc oxide or gallium phosphide) with very small spatial extension, with a very small pump spot, or with a stripe-shaped spot [11]. In particular it has been shown [12] that by increasing the pump spot of a RFRL a smooth spectrum is recovered.

Up to now different theoretical approaches have been suggested to account for the different aspects of RLs. The RL spectrum has been investigated through the strong nonlinear interaction of RL individual lossy modes [13], while in analogy with condensed-matter physics a spin-glass-like model [14] predicts the existence of various thermodynamic phases,

*marco.leonetti@icmm.csic.es including a mode-locked condition, in which lasing resonances are synchronized. A different approach, valid in the condition in which the number of resonances pushes to infinity, allows us to cast an equation for the spectral shape of the intensity, predicting the behavior as a function of energy, in agreement with the experiments [15].

This review follows a study [16] that has demonstrated that the particular regime (so far called either resonant or diffusive) in which the RL may appear is driven by the degree of mode synchronization. This has been demonstrated by following an experimental approach consisting of several features: (i) random lasing system, in which a single strong-scattering cluster is isolated and embedded in a dye solution, (ii) a pumping scheme in which the cluster is optically pumped by using stimulated emissions generated in the dye, thus allowing us to obtain directional pumping, and (iii) an engineered scheme to collect the RL spectrum.

Our approach allows us to tune and monitor the amount of activated lasing modes in a single micron-sized titanium dioxide cluster and to measure the intermode correlation and the transition from an uncorrelated (with a RFRL-like spectrum) to a strongly correlated random lasing (with an IFRL-like spectrum). In this paper we report extensive details on this experimental approach, including an analysis of the random laser distribution of the intensity.

\section{SAMPLE PREPARATION}

The majority of previous experiments have been performed by exploiting micron-size powder arranged in macroscopic (millimeter-size) samples. As demonstrated in a recent paper, the extension of random lasing modes may be as small as a few microns [17]; thus if one wants to study the properties of a small set of modes, the dimension of the system should be comparable. A possible strategy is the one used in the work by Fallert et al. [18] in which the spatial structure of random lasing modes has been studied in a compact cluster of zinc oxide powder. This material has sçeveral advantages: very high index contrast and very high damage threshold. Random lasing may be also achieved by using titanium dioxide dispersions in solutions containing rhodamine [3]; even if it is 


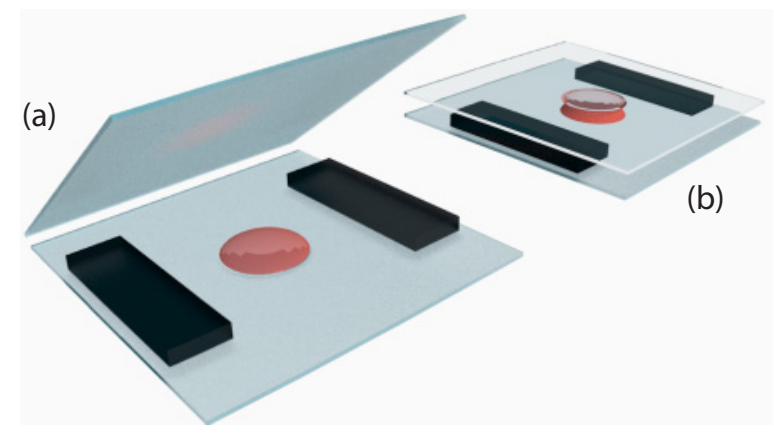

FIG. 1. (Color online) Sample preparation: (a) A drop of the solution containing scattering particles and dye is placed on a glass substrate with two plastic spacers. (b) A second substrate is placed on the top of the sample, squeezing the drop.

less efficient, this system possesses a fundamental difference from zinc oxide: gain and scattering are hosted by two different materials, and we exploit such a feature to achieve control over the modes. To obtain a micron-size cluster structure embedded in the rhodamine solution we proceed as follows: First, we produce a solution containing $1 \mathrm{vol} \%$ of titanium dioxide powder [titanium (IV) oxide, 89490 Sigma-Aldrich, particle size $\leqslant 1 \mu \mathrm{m}]$ in ethanol; then we produce another solution containing 1 vol \% of rhodamine B (Sigma-Aldrich 242425) in ethylene glycol. The first and the second solutions are mixed in equal parts to obtain a third solution that is placed in an ultrasound bath for half an hour. A single drop (from 2 to $5 \mu \mathrm{L}$ ) is placed on a thin microscopy coverslip [Fig. 1(a)]. After two plastic spacers (thickness $S=50 \mu \mathrm{m}$ ) are placed, the drop is squeezed with a second coverslip [Fig. 1(b)]. In this thin system we observed on the bottom glass the formation of clusters of titanium dioxide with a size of the order of tens of microns. Figure 2(a) shows images obtained with an optical microscope in transmission geometry of the sample in which the clusters appear as black spots in a rhodamine (pink) bath.

Several minutes after the sample fabrication the clusters appear to be completely formed and stable in their position and shape. Clusters possess random shapes and sizes and usually appear close together, a condition making the experimental study of an isolated cluster difficult. To obtain an isolated cluster we proceeded as follows: Once a target cluster has been selected we pump it with a beam from a Nd:YAG pulsed laser (fluence of $0.02 \mathrm{~nJ} / \mu \mathrm{m}^{2}$ ) by using the setup described below. The beam heats the liquid solution, generating a flux that slowly moves the other clusters away from the cluster placed in the center of the pump spot (we see the flux acting
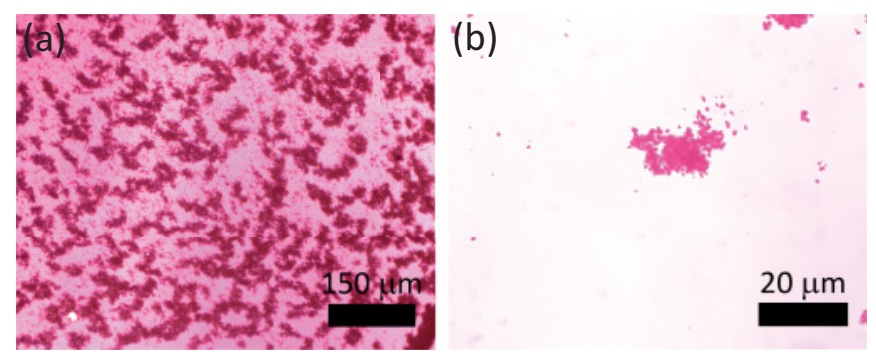

FIG. 2. (Color online) Sample (a) before and (b) after the isolation protocol.
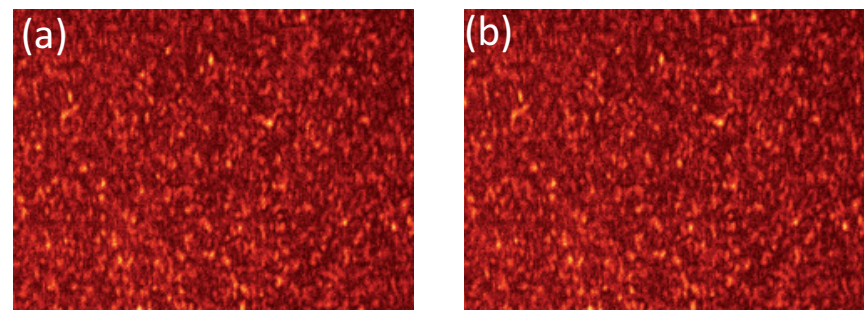

FIG. 3. (Color online) Passive speckle pattern from a single cluster obtained (a) before and (b) after 150 pump shots (Image size $16 \times 12 \mathrm{~mm}$ ). The correlation between the two images is 0.85 .

principally at the edges of the pumped region). Figure 2(b) shows a single cluster after this isolation protocol.

We observed by optical imaging that cluster positions remain fixed for days. The RLs analyzed below are critically dependent on cluster position; thus we further investigated the cluster stability by monitoring fluctuations in the speckle pattern obtained by shining a continuous helium-neon laser on a single cluster before and after 150 shots from the pump laser, following reference [7]. The two speckle patterns in Fig. 3 show a correlation of about 0.85 , denoting a static disorder.

\section{EXPERIMENTAL SETUP}

In this paper we will demonstrate that the distribution of the spectral peaks, the intermode correlation, and the spatial distribution of the intensity may be controlled by the pumping shape, which directly affects the interaction between resonances. The modes interact because the considered cavities are open, so that leaked radiation may travel between different modes of the disordered structure. This picture features a degree of interaction strongly affected by the distance between the modes, i.e., modes occurring close together will interact more strongly than modes that are at distant positions. In previous experiments peaks of the RFRL spectrum have been excited by a circularly focused beam. This represents a strong constraint in the study of RL properties: if all modes are activated in a small area, they necessarily stay close together, meaning their interaction is always strong. We developed a different approach that allows us to tune the degree of interaction between the modes (we call it directional pumping). In a standard RL experiment, energy is brought to the modes by an external laser pump so that the absorption and reemission process does not possess any specific direction. A directionality may be introduced by using a stripe-shaped pump beam in which a flux of stimulated emission is generated along the stripe direction. A similar approach, which has been already exploited to study diffusive systems [19], allows us to turn on modes that are much less interactive than the circular pumping configuration. In fact modes do not have the constraint to lie close to one another; instead, they only have to comply with the condition of being efficiently coupled to the input direction.

A scheme of the setup is given in Fig. 4. The pulse of the pump laser is generated by a frequency-doubled $\mathrm{Nd}$ :YAG pulsed laser, with a frequency of $532 \mathrm{~nm}$, maximum pulse energy of $125 \mathrm{~mJ}$, and pulse duration of $9 \mathrm{~ns}$. To achieve complete control over the shape of the pump beam we used a reflective spatial light modulator from Holoeye 


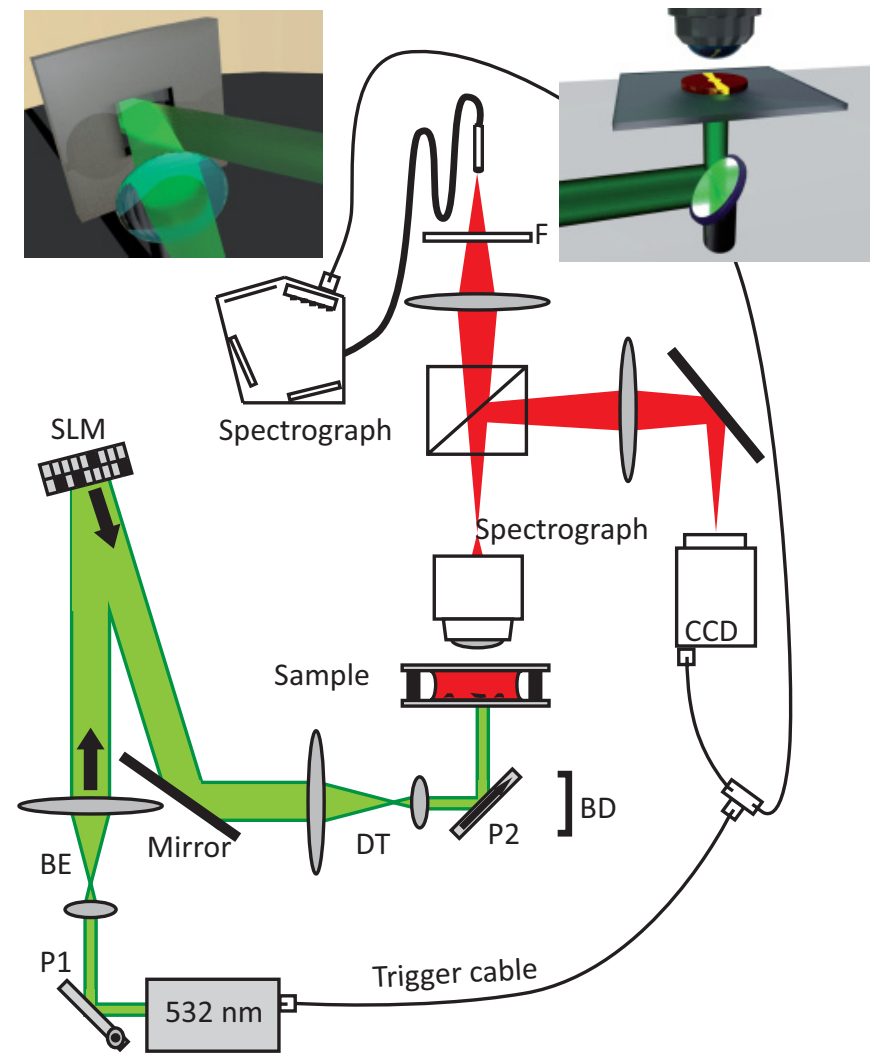

FIG. 4. (Color online) A sketch of the experimental setup. The vertically polarized laser beam is first reflected by a high-power dichroic polarizing mirror $(\mathrm{P} 1)$ to clean residual unpolarized light and then passes through a $5 \times$ beam expander $(\mathrm{BE})$ before impinging on the spatial light modulator (SLM). A demagnifying telescope (DT) images the spatial light modulator on the sample after a polarizing dichroic mirror (P2), which selects horizontal polarization, while residual vertically polarized light is absorbed by a beam dump (BD). Emitted light is collected by a microscope objective (numerical aperture of 0.40 , working distance of $6 \mathrm{~mm}$ ) to be imaged by an enlarging telescope $(10 \times)$ on a $50-\mu \mathrm{m}$-core fiber after being filtered by an edge filter $(\mathrm{F})$ cutting residual green laser light. RL light is divided by a beam splitter to feed a spectrograph and a CCD imaging camera, both of which are triggered by the pumping laser. The right inset shows an explicative perspective view of the SLM, while the left inset represents the sample under pumping.

(LC-R 1080, $1920 \times 1200$ pixels) in amplitude-modulation configuration [20]. Light emitted from the sample is collected by a microscopy objective and sent to a spectrograph and to an imaging camera by a 50:50 beam splitter. The spectrum is collected by a $50-\mu \mathrm{m}$ fiber placed in the image plane of an enlarging $(10 \times)$ telescope in transmission geometry, thus collecting light from a $5-\mu \mathrm{m}$ area at the center of the sample. A colored filter eliminates residual pump light. Spectra are obtained by a 303-mm focal length spectrograph (Andor, Shamrock 303) connected to a low-noise charge-coupled device array (Andor, iDus Spectroscopy CCD). An image of the sample is obtained with a standard scientific camera (Pixelink model PL-B776F).

\section{RANDOM LASER ACTIVATED BY DIRECTIONAL PUMPING}

In this section we will describe the properties of a directionally pumped random laser. We will study a single cluster of titanium dioxide embedded in a rhodamine solution. In this configuration the properties of the RL emission from the cluster depend not only on the energy that arrives directly from the external pump to the cluster but also on the energy arriving from the emission generated in the neighborhood of the cluster. This is demonstrated by monitoring the emission of a single cluster illuminated by a beam shaped in the stripe configuration (16- $\mu \mathrm{m}$ thickness) while varying the length of the long side of the stripe, which affects the intensity of the stimulated emission, which is the indirect pumping shining on the cluster. In Fig. 5 we show the intensity and full width at half maximum (FWHM) of the emission as a function of the length of the stripe shining on the sample.

The growth of the intensity measured when the stripe is longer may only be explained by the presence of indirect and directional pumping. The narrowing of the FWHM proves the onset of the lasing regimes at large values of the stripe length. We stress that our confocal configuration in which a fiber is placed in an enlarged image plane, allowing us to spatially filter all the light that does not arise from the cluster, is fundamental to measuring the described phenomena; otherwise, the fluorescence from the whole pumped area would hide the laser emission from the cluster.

Then we study different stripe configurations that all share the same length $(1.6 \mathrm{~mm})$ but have different orientation and thickness. In Fig. 6(a) (black solid line) we report the average spectrum of 100 acquisitions or "shots" from a pumping stripe with a thickness of $16 \mu \mathrm{m}$ (orientation thickness and pump power on the stripe have conditions that we identify as configuration 1). Noticeably, the presence of sharp (subnanometric) and repeatable peaks in the spectrum is connected to the appearance of bright spots in the spatial distribution of the intensity [Fig. 6(c)]; thus these features are due to modes dwelling in the cluster structure. Increasing the stripe thickness (32 $\mu \mathrm{m}$, configuration 2) produces a larger number of activated modes (Fig. 6, red dashed line), and

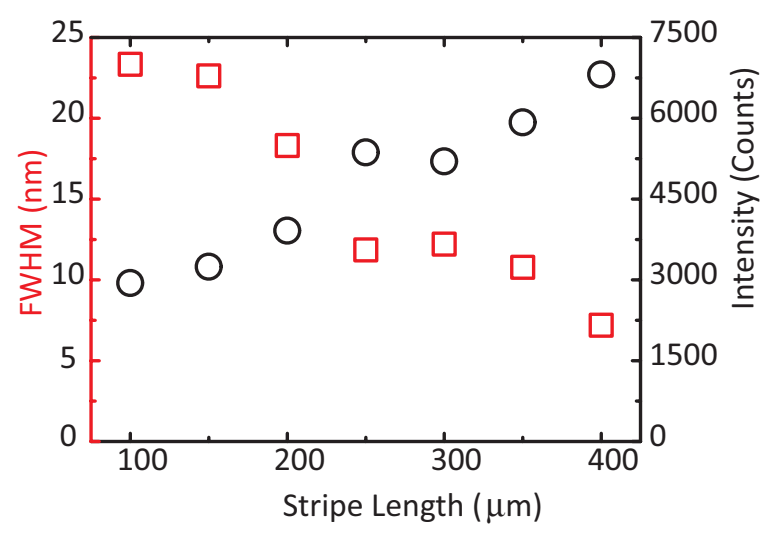

FIG. 5. (Color online) FWHM (red squares) and peak intensity (black circles) as a function of the length of the illuminated stripe. The measurement has been performed with a pump power of $0.1 \mathrm{~nJ} / \mu \mathrm{m}^{2}$ pulse. 

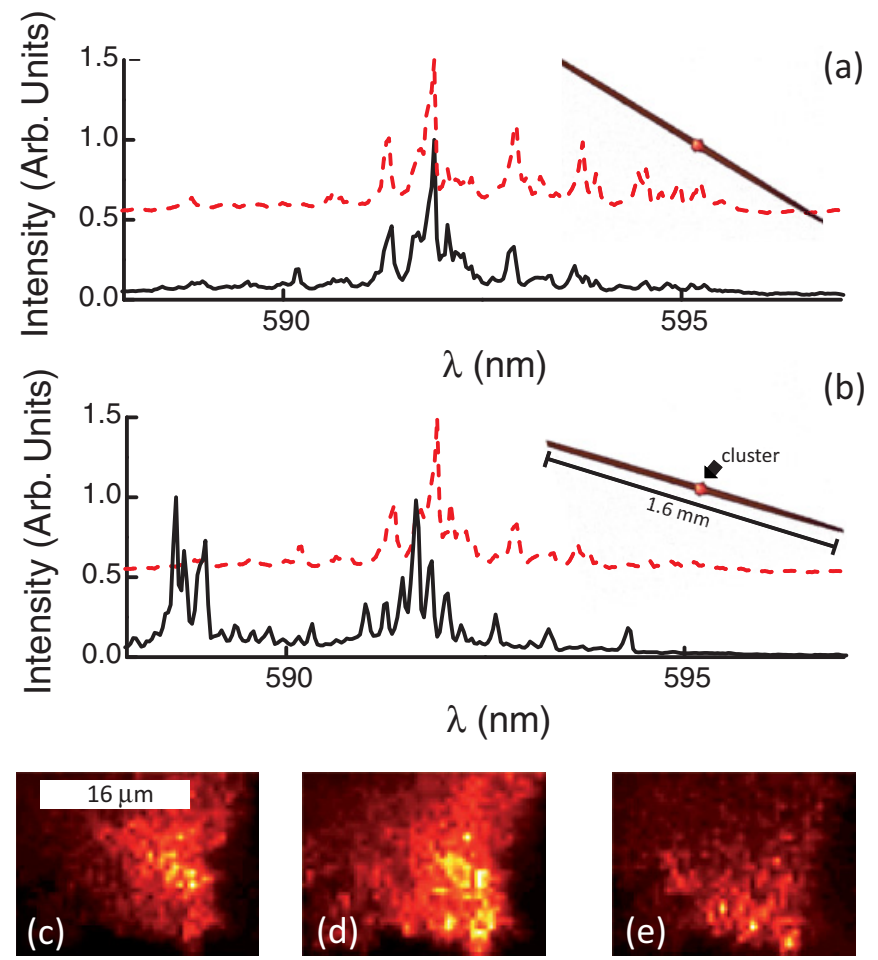

FIG. 6. (Color online) (a) Spectrum (solid line) obtained with configuration 1 (see text) together with the spectrum obtained with the same orientation but with a $32-\mu \mathrm{m}$-thick stripe (configuration 2, dashed red line arbitrarily translated along the ordinate). (b) Spectrum obtained by pumping with configuration 3 obtained by tilting the stripe by $15^{\circ}$ with respect to configuration 1 . The spectrum obtained from configuration 1 is reported as a dashed red line and arbitrarily translated along the ordinate for comparison. The spatial distribution of intensity for configurations (c) 1, (d) 2, and (e) 3 . The inset represents the pumping shape of configurations 1 and 3 . Al measurements are obtained with a $0.1 \mathrm{~nJ} / \mu \mathrm{m}^{2}$ pump fluence

new bright spots appear in the spatial distribution of intensity [Fig. 6(d)]. This particular set of activated modes is nearly unaffected by the stripe thickness, although it is strongly sensitive to the stripe orientation. The spectrum in Fig. 6(b) (solid black line) has been obtained for a 16- $\mu \mathrm{m}$-thick stripe tilted by $15^{\circ}$ (configuration 3 ) with respect to the previous measurement (reported for visual comparison by the red dashed line in the same panel). Figure 6(e) shows the relative spatial distribution of the intensity and that this $15^{\circ}$ tilt also affects the location where hot spots appear.

A different RL regime is obtained when directionality is absent, that is, when using a circular pump spot. In Fig. 7 we show the spectrum obtained from the same cluster as in Fig. 6 (we identify this particular cluster as C1) but using a circular pump with a 1-mm diameter (a sketch is given in the left inset of Fig. 7) for two different values of the pump fluence. The retrieved spectrum in this case appears as a linenarrowed smooth peak with tiny features (small peaks) that are repeatable from shot to shot. The spatial distribution of the intensity (left inset) appears smooth and lacks the bright spots that can be seen in the stripe case.

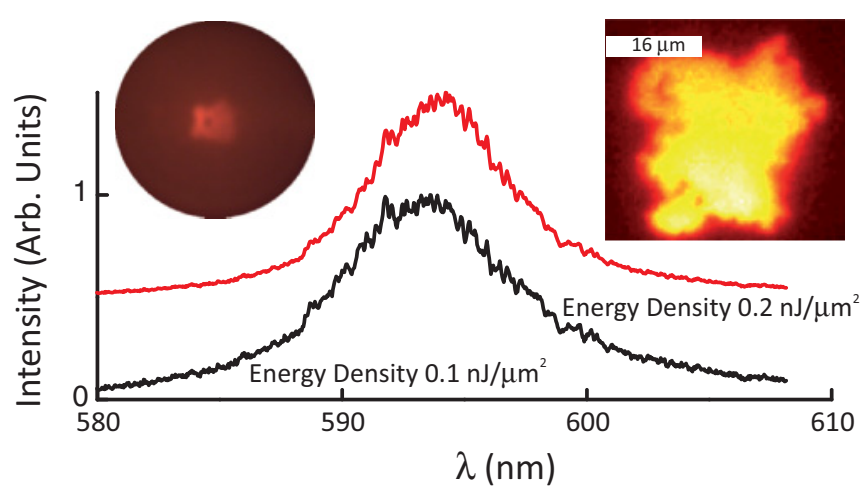

FIG. 7. (Color online) Spectrum obtained for two different values of the pump power with a circular pump spot (left inset) on the cluster in Fig. 6. An image of the intensity distribution of the cluster is shown in the right inset.

\section{TRANSITION FROM A SPIKY TO A SMOOTH RANDOM LASER}

With the previously reported measurements we recognized the possibility to shift lasing emission between two possible emission regimes. By using a stripe-shaped pump we retrieve a RFRL, while a circular pump results in an IFRL emission. In this section we demonstrate that it is possible, by controlling the pump shape, to turn gradually from one regime to the other and control the degree of "spikiness" of the spectrum.

To this aim we engineered a more complex pumping geometry: the pie-shaped pumping (Fig. 8). The excited area consists of a disk ( $150 \mu \mathrm{m}$ in diameter) centered on the cluster (to assure homogeneous pumping even for the largest clusters) to which two symmetrical wedges with a much larger radius (1 $\mathrm{mm}$ in diameter) and controllable orientation and aperture angle $(\Theta / 2)$ are added, which serve as launch pads for the directional stimulated emission. A single-wedge configuration led to similar results but proved to be hydrodynamically less stable. The central circle places the cluster barely below the lasing threshold, preparing the system for lasing once the wedges are turned on. $\Theta$ controls the angular aperture with which the stimulated emission is produced and therefore controls the number of modes expected to be excited.

We note that the number of modes is strongly affected by the $\Theta$ parameter: in Fig. 9(a) we plot the number of peaks in the spectrum $N$ (that is, the number of local maxima of the

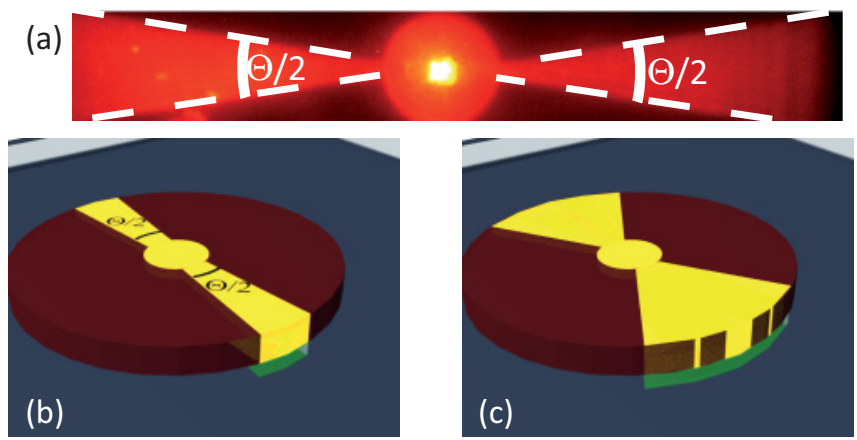

FIG. 8. (Color online) (a) An image of the sample shined with the pie-shaped pumping. (b) and (c) A perspective scheme of the pumped sample for two different values of $\theta$. 

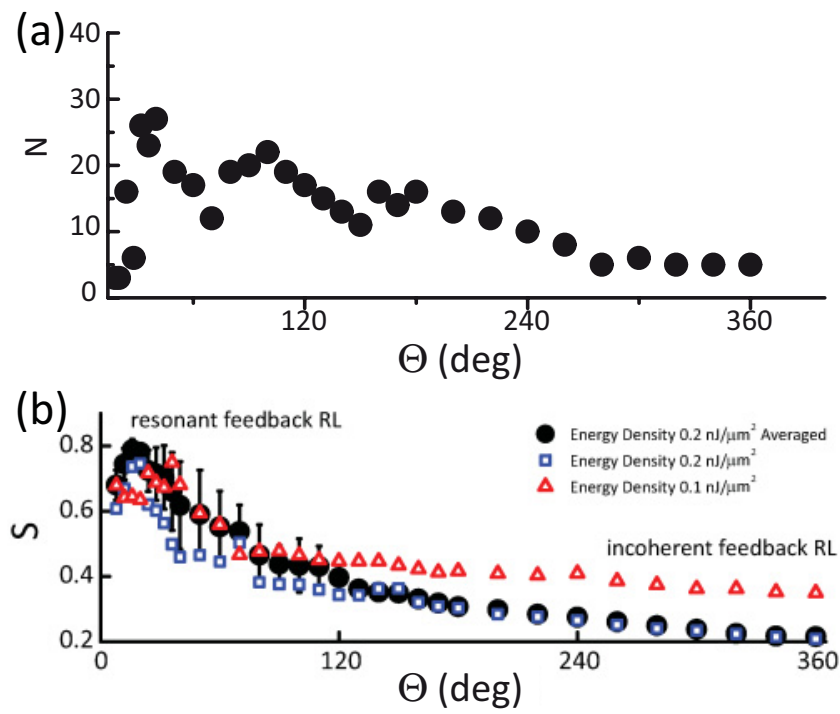

(c)

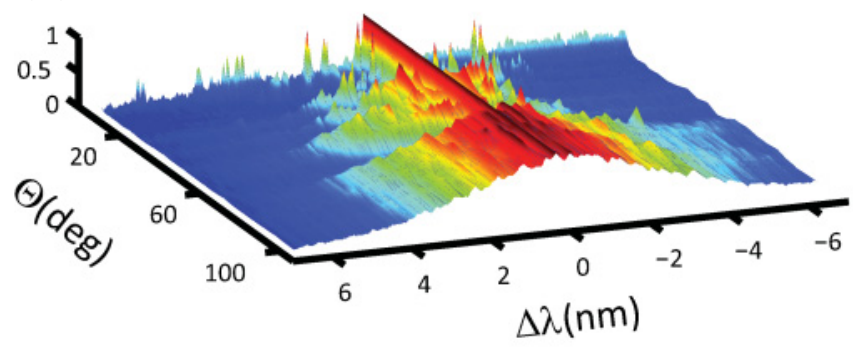

FIG. 9. (Color online) (a) Number of modes $N$ and (b) spikiness as a function of $\Theta$. Squares and triangles correspond to different pump energies for cluster $\mathrm{C} 1$. Circles (error bars) corresponds to the average (standard deviation) of five measurements from different clusters. (c) Three-dimensional graph showing normalized spectra (average over 100 shots with a fluence of $0.2 \mathrm{~nJ} / \mu \mathrm{m}^{2}$ ) for different $\Theta$. Spectra are arbitrarily shifted in frequency to superimpose intensity maxima. $\Delta \lambda$ is the wavelength shift from the most intense peak.

spectrum measured after a smoothing has been performed to eliminate noise effects) as a function of $\Theta$. The number of modes in the spectrum first grows because the increased width of the input directions allows for the excitation of more modes, and then it decreases because multiple peaks merge into a single narrowed IFRL. To classify a RL into the IFRL or RFRL categories we measure its spikiness $S$, which is the number of high-frequency components of the Fourier transform (FT) of the emission. We define $S$ as the high-frequency fraction of the total FT area, i.e., the area above the frequency threshold. As a cutoff we defined $K=1.20 \mathrm{~nm}^{-1}$ in the horizontal scale of the FT, and then we calculated $S$ as the area of the FT lying in the high-period part with respect to a threshold $K$ (i.e., corresponding to periods greater than $K$ ). $S$ returns a value close to 1 for very spiky spectra, while a value close to 0 is retrieved for smooth spectra.

Figure 9(b) shows $S$ versus $\Theta$ for two pump energies (squares and triangles) for cluster $\mathrm{C} 1$ and averaged over five different clusters (circles). All curves display the same trend, evidencing a transition: after a rapid growth corresponding to an increase in fluence and number of excited modes (appearing on a smooth fluorescence spectrum), $S$ reaches a maximum
(RFRL regime), after which the spectrum becomes smoother as $\Theta$ grows until an IFRL-like emission is achieved. In Fig. 9(c) we show the evolution of the average spectra from the spiky case to the smooth case. Note that smoothing at high $\Theta$ is not due to averaging because sharp peaks are absent in single-shot spectra too.

\section{CORRELATION OF THE RL FLUCTUATIONS}

In our experiments both IFRL and RFRL show intensity fluctuations from shot to shot, while the mode (spectral) positions remain unchanged. Nevertheless, the properties of the fluctuations of a RFRL strongly differ from that of an IFRL. In particular for a RFRL intensity fluctuations are uncorrelated (i.e., modes oscillate independently, that is, one mode can grow in intensity while the others decrease), while for an IFRL a decrease (increase) in the intensity at one frequency is strongly correlated to the trend of the intensity for all the other modes.

Let us consider the intensity of two modes of a RFRL [spectrum shown in Fig. 10(a) obtained with $\Theta=18^{\circ}$ ] and of an IFRL [spectrum shown in Fig. 10(b)] obtained with $\Theta=$ $360^{\circ}$ from the same sample (cluster C1) with two different pumping. In Fig. 10(c) we plot 100 values of the intensity obtained for 100 single-shot measurements in the small $\Theta$ configuration. The correlation between the two intensity ensembles is calculated by using the Pearson correlation defined as follows:

$$
C_{p}=\frac{\sum_{i=1}^{N}\left[I\left(\lambda_{1}\right)_{i}-\bar{I}\left(\lambda_{1}\right)\right]\left[I\left(\lambda_{2}\right)_{i}-\bar{I}\left(\lambda_{2}\right)\right]}{\sqrt{\sum_{i=1}^{N}\left[I\left(\lambda_{1}\right)_{i}-\bar{I}\left(\lambda_{1}\right)\right]^{2}} \sqrt{\sum_{i=1}^{N}\left[I\left(\lambda_{2}\right)_{i}-\bar{I}\left(\lambda_{2}\right)\right]^{2}}},
$$

where $\bar{I}$ represents the average over the ensemble. If two modes show $C_{p}$ close to 0 , their fluctuations are uncorrelated, while correlated modes result in $C_{p}$ close to 1.
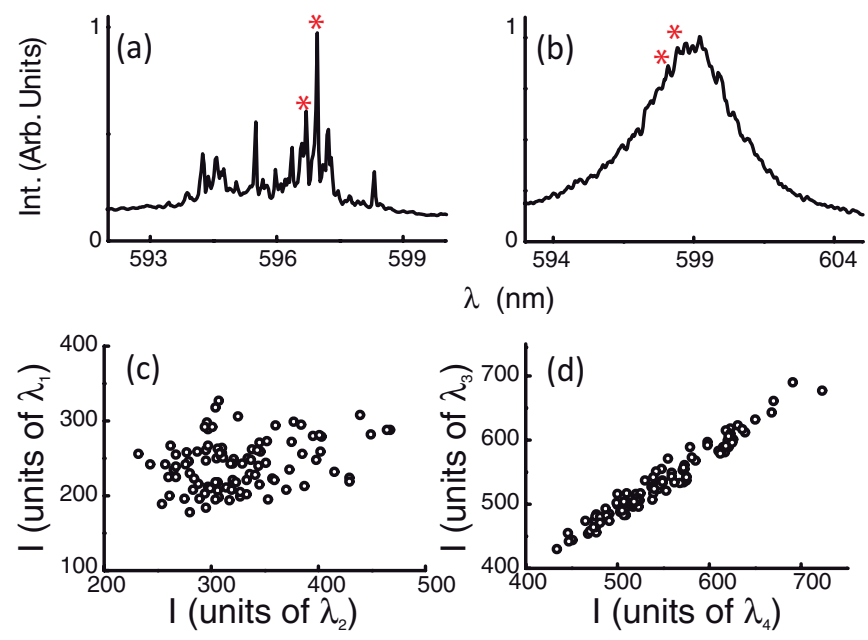

FIG. 10. (Color online) Normalized average spectra from cluster $\mathrm{C} 1$ for $(\mathrm{a}) \Theta=18^{\circ}$ and $(\mathrm{b}) \Theta=360^{\circ}$. Asterisks indicate the positions of the modes analyzed in (c) and (d). (c) Intensity values of the modes at wavelengths $\lambda_{1}=597.2 \mathrm{~nm}$ and $\lambda_{2}=596.7 \mathrm{~nm}$ obtained for 100 single-shot realizations in the pumping configuration with $\Theta=18^{\circ}$. (d) As in (c) for wavelengths $\lambda_{3}=598.4 \mathrm{~nm}$ and $\lambda_{4}=598.7 \mathrm{~nm}$ and $\Theta=360^{\circ}$. 

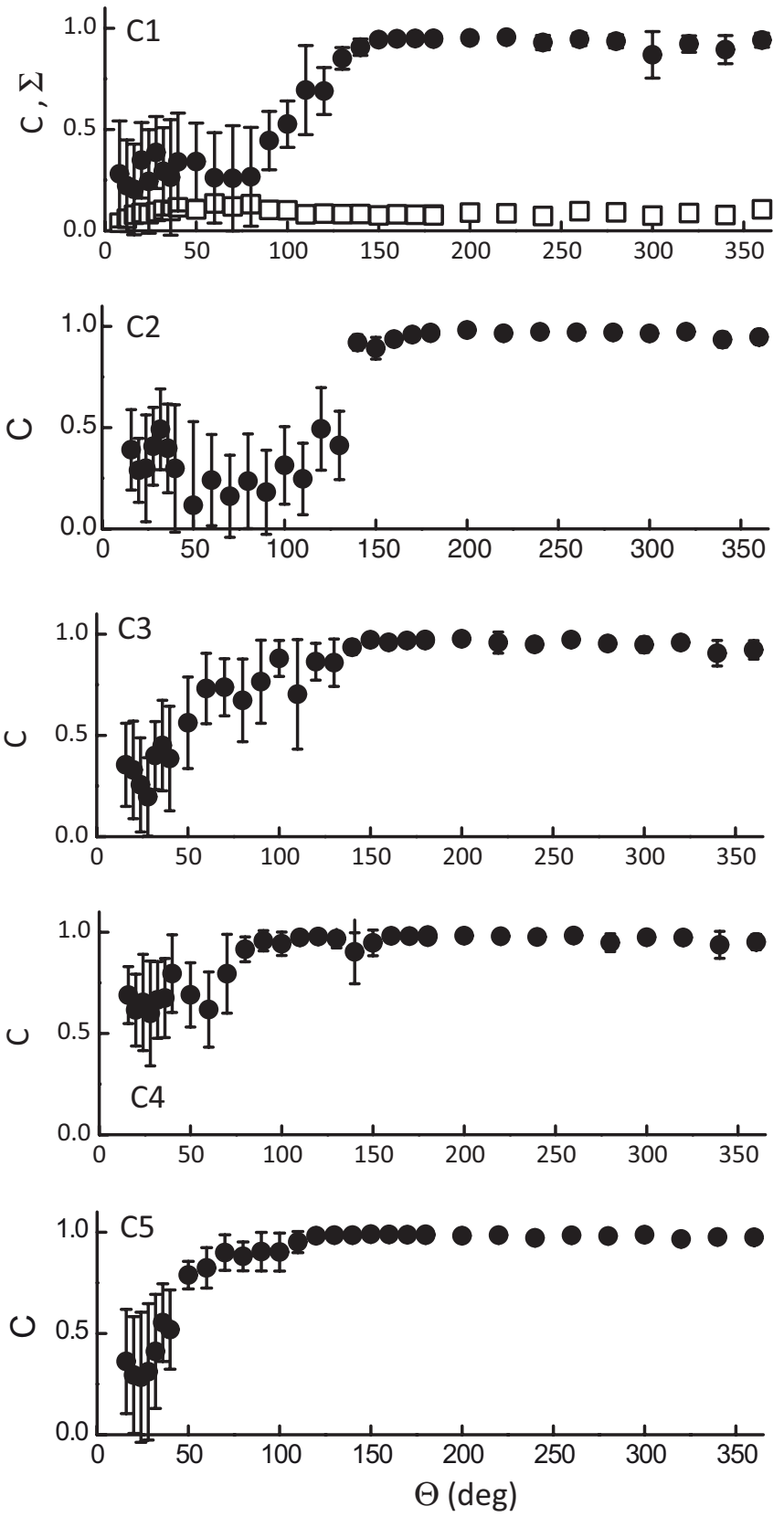

FIG. 11. Average correlation $\mathrm{C}$ as a function of $\Theta$ obtained for five different clusters. In the graph for $\mathrm{C} 1, \Sigma$ is also plotted as a function of $\Theta$ (open squares).

The correlation for the modes in Fig. 10(a) is 0.47, while for the modes in Fig. 10(b) the correlation is 0.97 . Then we calculate the average correlation $C$ (Fig. 11) as the average of $C_{p}$ over 105 wavelength pairs, corresponding to the 15 most intense peaks, demonstrating that modes of an IFRL are, on average, much more correlated than that of a RFRL.

Figure 11 shows the average correlation $C$ versus $\Theta$ for five different clusters, named $\mathrm{C} 1$ to $\mathrm{C} 5$. The onset of a strongly correlated regime is obtained for $\Theta \cong 120^{\circ}$. The fact that the same transition has been observed in all the considered samples reveals a universal trend in which $C>0.8$ when $\Theta>180^{\circ}$. The reported behavior of $C$ at large $\Theta$ is due to interaction and not to a lowering of the overall fluctuations. This is demonstrated

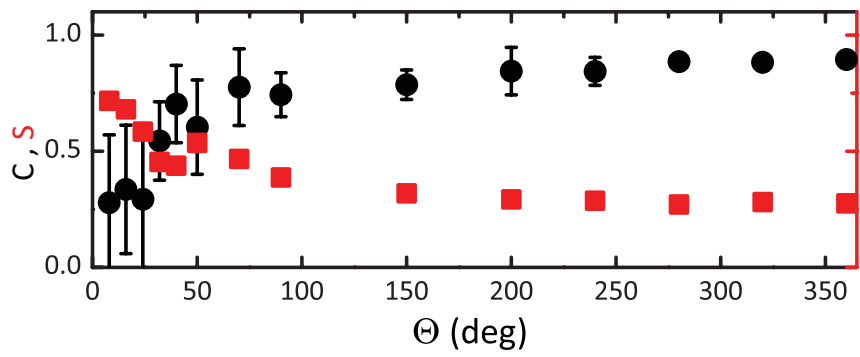

FIG. 12. (Color online) $C$ (circles) and $S$ (squares) as a function of $\Theta$ in the fixed volume configuration. Pumping is $0.1 \mathrm{~nJ} / \mu \mathrm{m}^{2}$

in the graph for $\mathrm{C}$, which shows $C$ together with relative fluctuation $\Sigma$ defined as $\sigma(\lambda) / \overline{I(\lambda)}$ [where $\sigma(\lambda)$ is the standard deviation of the intensity for the frequency $\lambda$ over 100 shots] averaged for the 15 most intense modes of cluster $\mathrm{C} 1$. The fact that $\Sigma$ is nearly constant for all values of $\Theta$ investigated allows us to exclude artifacts.

\section{MEASUREMENT AT FIXED VOLUME}

The parameter that controls the transition between uncorrelated and correlated random lasing is the angle $\Theta$, which defines the possible input directions on the cluster. To increase $\Theta$ we add to the inverted area angular sections of a disk. Although this choice clearly defines the input directions, a variation of $\Theta$ does not conserve the volume of the illuminated part of the sample and the total energy impinging on the sample. To confirm that the change in regime is due to the quality of the intermode interaction, we performed a measurement at fixed volume and energy. The measurement is very similar to the previous one with a central disk plus the angular sectors of a larger circumference, but in this case for every value of $\Theta$ we also adjusted the value of the radius $R$ of the external circumference in order to conserve the area of the illuminated surfaces. In practice an increment of $\Theta$ corresponds to a decrease in the value of $R$ to preserve a fixed total laser spot of $0.06 \mathrm{~mm}^{2}$. Figure 12 shows the relative measurement of $C$ and $S$ as a function of $\Theta$ for this fixed volume configuration. This measurement allows us to rule out the effects of volume- or energy-dependent terms, such as amplified spontaneous emission.

It is also worth mentioning that $S$ and $C$ do not depend on how many wedges are used to compose the pumping area. Although we show results for only two wedges, any number can be used with similar results.

\section{SPATIAL PROPERTIES OF THE SPATIAL DISTRIBUTION OF INTENSITY}

It was shown in a previous section (Figs. 6 and 7) that the spatial distribution of the intensity is strongly affected by $\Theta$ : it is characterized by a profile with sharp features (hot spots) in the RFRL case, while it displays a smooth spatial profile for large $\Theta$ [see Fig. 13(a)]. Here we investigate this feature by studying the inverse participation ratio $P$ of the intensity distribution, which quantifies the degree of confinement of the 

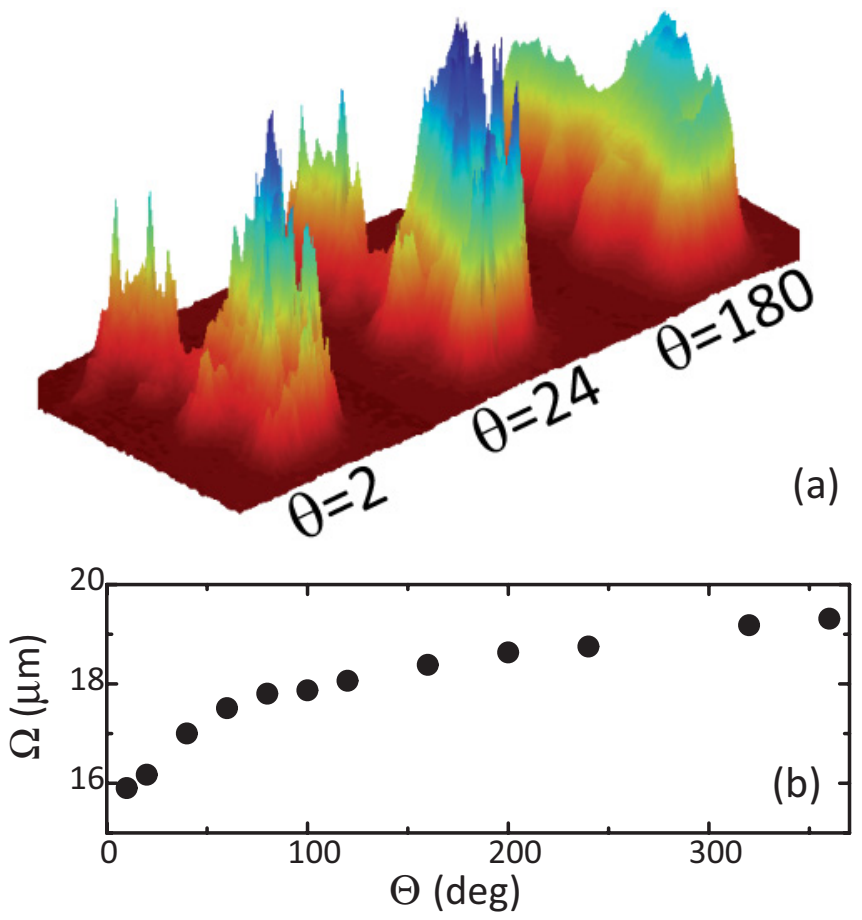

FIG. 13. (Color online) (a) Surface plot of the intensity pattern for the same cluster for three different values of $\Theta$. (b) $\Omega$ plotted as a function of the parameter $\Theta$.

light in the sample [21,22]. It may be calculated as

$$
P=\frac{\int I^{2}(x, y) d x d y}{\left[\int I(x, y) d x d y\right]^{2}},
$$

where $I(x, y)$ is the measured spatial intensity distribution of the light. From $P$, which has the units of an inverse area, one may obtain the extension $\Omega=\left\langle P^{\frac{-1}{2}}\right\rangle$, which has the dimension of length. In Fig. 13(b) $\Omega$ is plotted as a function of the parameter $\Theta$. The the presence of sharp features for an intensity spatial distribution organized in a more localized fashion is a signature of the fact that energy is stored in particular points of the sample and may not travel for one place to the other for low $\Theta$, in agreement with the low values of $C$ measured for $\Theta<100^{\circ}$. Conversely, at high $\Theta$, a larger number of modes is activated, and consequently, the whole cluster is filled with activated resonances, and energy may flow through the cluster, resulting in a smooth spatial profile and a more correlated emission, consistent with an increased intermode interaction.

\section{TIME-RESOLVED MEASUREMENTS}

The reported behavior of $C$ and $S$ may also be obtained with a picosecond pump pulse (we used a 30-ps pulse frequency-doubled Nd:YAG laser with $60 \mathrm{~mJ} /$ pulse maximum energy), which also allows us to resolve temporal behavior of emitted light [23]. Light is collected from a single cluster by a photodiode (1-ns rise time and 1-ns fall time) and a 300-MHz oscilloscope. In Fig. 14 we show results for cluster C1. Lasing in the correlated regime $\left(\Theta=140^{\circ}\right)$ shows shorter pulses with respect to the uncorrelated $\left(\Theta=18^{\circ}\right)$ random laser for all five clusters. We obtained an estimate of the RL pulse duration by measuring the full width at half maximum of the peak

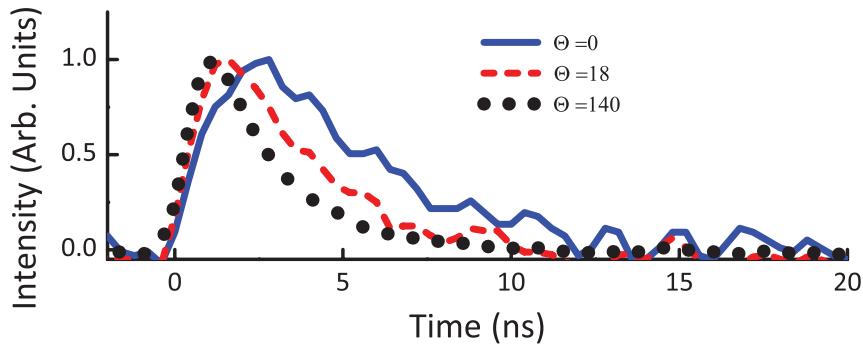

FIG. 14. (Color online) Intensity as a function of time for cluster $\mathrm{C} 1$ pumped with wedges of different aperture. Measurements are performed at a pump fluence of $0.1 \mathrm{~nJ} / \mu \mathrm{m}^{2}$.

in intensity of the average of eight single-shot time-domain measurements, retrieving an average shortening of about $30 \%$ for the IFRL with respect to the RFRL. In Fig. 14 we also report the temporal shape of the fluorescence obtained by pumping the sample only with the central disk $\left(\Theta=0^{\circ}\right)$.

In the ordered case, pulse duration scales as $1 / N$ [24]; conversely, in the disordered case it is not at all expected that the pulse duration will decrease substantially with the number of modes, but it is reasonable that some shortening occurs because the modes act synchronously, e.g., they tend to turn at the same time, because as one of them gets energy, it also feeds the others. We plan to study further all the aspects of mode locking in disordered structures in future theoretical and numerical works.

\section{ORIGIN OF PHASE LOCKING IN RANDOM LASERS}

In this section we wish to deepen the physical interpretation of the numerical approach discussed in Ref. [16] that demonstrates that the experimentally measured phenomena are due to the onset of a mode-locking regime. Historically, active mode locking has been the first approach pursued experimentally, in which the coupling between orthogonal modes is obtained by adding into the cavity an active optical element which introduces a modulation of strength $M$ with frequency $\Omega_{m}=2 \pi / T_{r}$, where $T_{r}$ is the round-trip time. The master equation for the amplitude of a mode propagating in such a cavity is the well known [25]

$$
\frac{\partial a(t)}{\partial t}=(g-\ell) a(t)+\frac{g}{\Omega_{g}^{2}} \frac{\partial^{2}}{\partial t^{2}} a(t)+M \Omega_{m}^{2} t^{2} a(t)-\gamma_{g}|a|^{2} a(t) .
$$

The first linear term represents the balance between gain $g$ and losses $\ell$, the second term derives from the finite bandwidth of gain approximated with a Lorentzian (width $\Omega_{g}$ ), the third term takes into account the external modulation, and the fourth represents the saturation of gain. This second-order differential equation dictates exponential solutions for any bounded pulse and shows stable solutions only if the gain saturation is included. It has been recently shown [15] that, in the limit in which the number of modes is very high (that is, the case of a macroscopic sample), the spectral shape of amplitude obeys an equation formally identical to Eq. (3), and the Schwalow-Townes-like behavior predicted by this approach has been experimentally measured. In our experiment, instead, the number of activated resonances is 
(a)
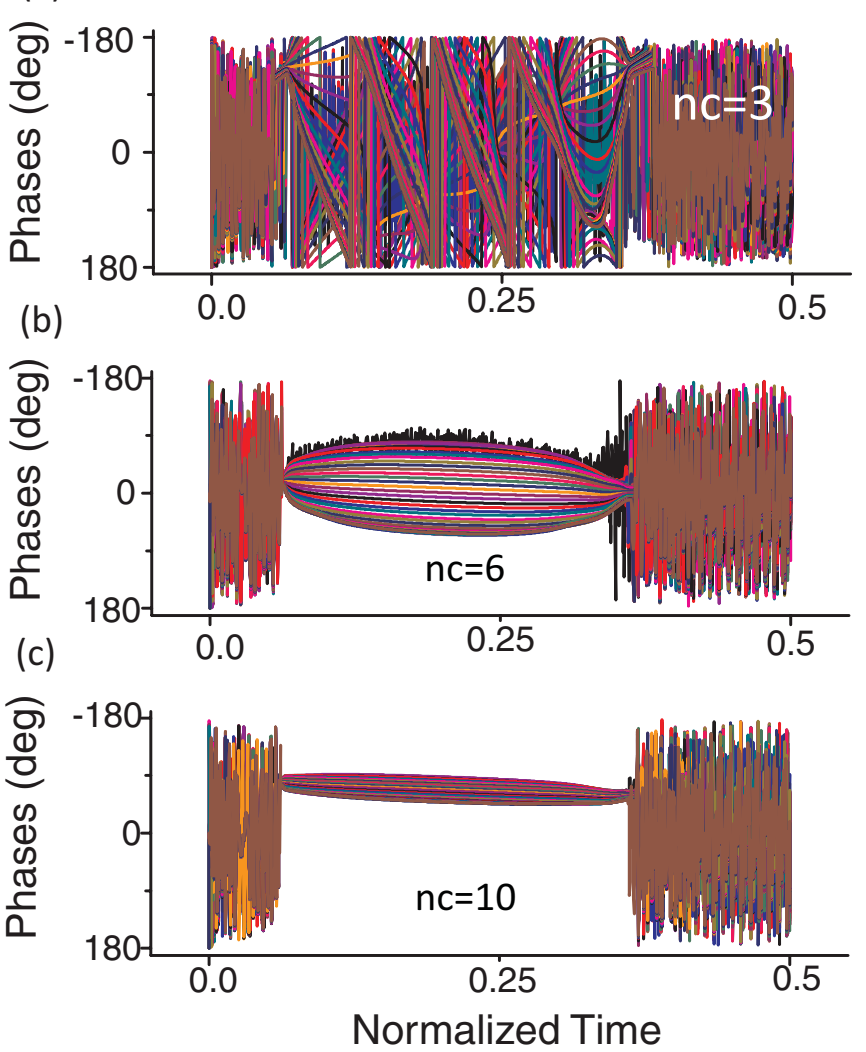

FIG. 15. (Color online) Results from numerical simulations. Temporal evolution of phases for different degrees of intermode coupling. $N_{c}$ is (a) 3, (b) 6, and (c) 10 .

limited both due to the pumping condition and to the micronsize extension of the clusters. Indeed we need to provide a different approach in which a discrete set of coupled modes is treated. The field amplitude in a micro-RL is sustained by several electromagnetic resonances, which are defined by their lifetimes and frequencies. Their amplitudes obey the coupledmode-theory equations (see [26] and references therein):

$$
\frac{d a_{n}}{d t}=+i \delta_{n} a_{n}-\alpha_{n} a_{n}+\sum_{m \neq n} \kappa_{n, m} a_{m}+g\left(\delta_{n}\right) \frac{a_{n}}{1+\gamma_{n}\left|a_{n}\right|^{2}},
$$

in which the resonant frequencies and their losses are given respectively by the frequency shift $\delta_{n}=\omega_{n}-\omega_{0}$ and the inverse decay times $\alpha_{n}$.

The linear gain linewidth can be approximated by a parabolic profile:

$$
g(\delta)=g_{0}(t)\left[1-\left(\frac{\delta}{\delta_{g}}\right)^{2}\right],
$$

where $g_{0}$ is the gain at the reference frequency $\omega_{0}$, taken at the peak of the fluorescence signal and corresponding to $\delta=0$, while $\delta_{g}$ measures the gain bandwidth. The gain saturation is included in the term containing $\gamma_{n}$, while the coefficients $\kappa_{n, m}$ represent the intermode coupling.

Note that, although Eqs. (3) and (4) share contributions with the same physical origin, the mechanism underlying the mode coupling is different. In standard laser cavities, modes are orthogonal and born uncoupled; thus it is necessary to use an external device that, by introducing a modulation synchronized with the round-trip time, produces sidebands for each mode that allows each one to be coupled with its nearest neighbors. The well-known solution of (3) is found analytically and is a Gaussian-shaped pulse whose duration may be compressed by increasing the coupling strength or the bandwidth of gain.

On the other hand, random lasing is sustained by (nonorthogonal) "quasimodes" that, being radiative, are naturally coupled and subject to a certain mode repulsion [27]. The randomness of coupling strengths makes it impossible to find a simple analytic solution for Eq. (4). Mean-field approaches (valid in the limit of a huge number of modes) allow us to predict the existence of distinct phases, including a mode-locked one [26]. In Ref. [16] we reported the temporal evolution of 50 modes obtained by numerically solving (4). While phase locking is clearly seen when the coupling strength $N_{c}$ increases (see Fig. 15), no effective pulse compression is found in the simulations. The relatively small compression of the pulse reported in a previous section may be thus ascribed to the synchronization of the onset of the lasing.

\section{CONCLUSIONS}

We described how to exploit directional pumping to control the set of activated modes and to onset different random lasing regimes. The spatial analysis reported here confirms the passage from a fragmented to a homogeneous landscape of the spatial distribution of the intensity, and it is compatible with an increase of the interaction at large $\Theta$. Moreover the mechanism underlying the phase-locking process has been deepened and compared with standard active mode locking. In our opinion the results reported here will open the way to a deeper understanding of the behavior of light in nonlinear and disordered systems, representing also the possibility of obtaining mode-locked random resonators and the fabrication of a new generation of all optically controlled photonic microdevices, thus stimulating theoretical and numerical analysis on the mode-locking process.

\section{ACKNOWLEDGMENTS}

This work was supported by ERC Grant (FP7/2007-2013) No. 201766, CINECA, EU FP7 NoE Nanophotonics4Enery Grant No. 248855, the Spanish MICINN CSD2007-0046 (Nanolight.es), MAT2009-07841 (GLUSFA), and Comunidad de Madrid S2009/MAT-1756 (PHAMA).
[1] V. Letokhov, Zh. Eksp. Teor. Fiz. 53, 1442 (1967).

[2] C. Gouedard, D. Husson, C. Sauteret, F. Auzel, and A. Migus, J. Opt. Soc. Am. B 10, 2358 (1993).
[3] N. M. Lawandy, R. M. Balachandran, A. S. L. Gomes, and E. Sauvain, Nature (London) 368, 436 (1994).

[4] D. S. Wiersma, Nat. Phys. 4, 359 (2008). 
[5] D. S. Wiersma and A. Lagendijk, Phys. Rev. E 54, 4256 (1996).

[6] S. Lepri, S. Cavalieri, G.-L. Oppo, and D. S. Wiersma, Phys. Rev. A 75, 063820 (2007).

[7] S. Mujumdar, V. Türck, R. Torre, and D. S. Wiersma, Phys. Rev. A 76, 033807 (2007).

[8] H. Cao, Y. G. Zhao, S. T. Ho, E. W. Seelig, Q. H. Wang, and R. P. H. Chang, Phys. Rev. Lett. 82, 2278 (1999).

[9] H. Cao, J. Y. Xu, D. Z. Zhang, S.-H. Chang, S. T. Ho, E. W. Seelig, X. Liu, and R. P. H. Chang, Phys. Rev. Lett. 84, 5584 (2000).

[10] K. L. van der Molen, R. W. Tjerkstra, A. P. Mosk, and A. Lagendijk, Phys. Rev. Lett. 98, 143901 (2007).

[11] H. Cao, Y. G. Zhao, H. C. Ong, and R. P. H. Chang, Phys. Rev. B 59, 15107 (1999).

[12] R. G. S. El-Dardiry, A. P. Mosk, O. L. Muskens, and A. Lagendijk, Phys. Rev. A 81, 043830 (2010).

[13] H. E. Tureci, L. Ge, S. Rotter, and A. D. Stone, Science 320, 643 (2008).

[14] L. Angelani, C. Conti, G. Ruocco, and F. Zamponi, Phys. Rev. Lett. 96, 065702 (2006).

[15] C. Conti, M. Leonetti, A. Fratalocchi, L. Angelani, and G. Ruocco, Phys. Rev. Lett. 101, 143901 (2008).
[16] M. Leonetti, C. Conti, and C. Lopez, Nat. Photonics 5, 615 (2011).

[17] K. L. van der Molen, R. W. Tjerkstra, A. P. Mosk, and A. Lagendijk, Phys. Rev. Lett. 98, 143901 (2007).

[18] J. Fallert, R. J. B. Dietz, J. Sartor, D. Schneider, C. Klingshirn, and H. Kalt, Nat. Photonics 3, 279 (2009).

[19] M. Leonetti and C. López, Opt. Lett. 36, 2824 (2011).

[20] N. Savage, Nat. Photonics 3, 170 (2009).

[21] S. Gentilini, A. Fratalocchi, L. Angelani, G. Ruocco, and C. Conti, Opt. Lett. 34, 130 (2009).

[22] T. Schwartz, G. Bartal, S. Fishman, and M. Segev, Nature (London) 446, 52 (2007).

[23] M. Siddique, R. R. Alfano, G. A. Berger, M. Kempe, and A. Z. Genack, Opt. Lett. 21, 450 (1996).

[24] A. Yariv, Quantum Electronics (Saunders College, San Diego, 1991).

[25] H. Haus, IEEE J. Sel. Top. Quantum Electron. 6, 1173 (2000).

[26] L. Angelani, C. Conti, G. Ruocco, and F. Zamponi, Phys. Rev. B 74, 104207 (2006).

[27] H. Cao, X. Jiang, Y. Ling, J. Y. Xu, and C. M. Soukoulis, Phys. Rev. B 67, 161101 (2003). 